\title{
Optical design of a spectrometer of high performance for planetary exploration
}

\section{Diseño óptico de un espectrómetro de altas prestaciones para la exploración planetaria}

\author{
M. Fernández-Rodríguez ${ }^{1 *}$, T. Belenguer ${ }^{1}$, M. Colombo $^{1}$, J. Cabrero ${ }^{2}$ \\ 1. Instituto Nacional de Técnica Aeroespacial (INTA)-28850 Torrejón de Ardoz-Madrid \\ 2. ISDEFE as external consultant for INTA-Ingeniería de Sistemas para la Defensa de España-28040-Madrid \\ $\left(^{*}\right)$ E-mail: fernandezrm@inta.es
}

Received: 13/07/2018 Accepted: 19/02/2019

DOI: $10.7149 /$ OPA.52.1.51007

\begin{abstract}
:
It is presented the optical design of a visible compact spectrometer for the analysis of the elemental and geochemical composition of Mars ground. The spectrometer works in the spectral range from 533nm to $676 \mathrm{~nm}$ achieving a spectral resolution of $0.14 \mathrm{~nm} / \mathrm{px}$. The challenge of this design resides in assuring the instrument performance under demanding environmental conditions of operation (operative temperature from $-40^{\circ} \mathrm{C}$ to $+6^{\circ} \mathrm{C}$ and compatible with space radiation) and, at the same time, with very rstrictive conditions of mass and volume.
\end{abstract}

Key words: spectrometer, Mars, Raman

RESUMEN:

Se presenta el diseño óptico de un espectrómetro de visible compato dedicado al análisis de la composición elemental y geoquímico del terreno de Marte. Trabaja en el rango espectral de $533 \mathrm{~nm}$ a $676 \mathrm{~nm}$ con una resolución media de $0.14 \mathrm{~nm} / \mathrm{px}$. El reto de este diseño reside en asegurar las prestaciones del instrumento en las exigentes condiciones ambientales de operación (temperatura operativa de $-40^{\circ} \mathrm{Ca}+6^{\circ} \mathrm{C}$ y compatible con alta radiación espacial) y , a su vez, con condiciones muy restrictivas de masa y volumen.

Palabras clave: espectrómetro, Marte, Raman

\section{REFERENCES AND LINKS / REFERENCIAS Y ENLACES}

[1] F. Rull, A. Sansano, E. Díaz, C. P. Canora, A. G. Moral, C. Tato, M. Colombo, T. Belenguer, M. Fernandez, J. A. R. Manfredi, R. Canchal, B. Dávila, A. Jiménez, P. Gallego, S. Ibarmia, J. A. R. Prieto, A. Santiago, J. Pla, G. Ramos, C. González, "ExoMars Raman laser spectrometer overview”. Proc. SPIE 7819, Instruments, Methods and Mission for Astrobiology XIII, 781915 (2010).

[2] C. V. Raman, K. S. Krishnan, "A new type of secondary radiation". Nature, 121, 501 (2018).

[3] P. Gallego, M. Fernández, V. Guembe, G. Ramos, C. Gonzalez, J. A. R. Prieto, R. Canchal, M. Colombo, T. Belenguer, P. Zuluaga, E. Diaz, A. Moral, F. Rull, "Transmission Grating Validation and Qualification Mars and Future Planetary Exploration Missions". European Planetary Science Congress, EPSC2013980-1, 8, (2013).

[4] https://sci.esa.int/sci-ft/50124-technology-readiness-level/

[5] M. Fernández Rodríguez, De los efectos del ambiente especial en las propiedades ópticas de vidrios y recubrimientos para sistemas espaciales, INTA 1st Ed. (2017), capítulo 7.

[6] https://wasatchphotonics.com 
[7] J. Smith, Modern Optical Engineering, SPIE Press 4th Ed. (2007).

[8] R. H. Czichy. "Optical design and technologies for space instrumentation". SPIE 2210, 420 (1994).

[9] C. P. Canora, A. G. Moral, F. Rull, S. Maurice, I. Hutchinson, G. Ramos, G. Lopez-Reyes, T. Belenguer, R. Canchal, J. A. R. Prieto, P. Rodriguez, P. Santamaria, A. Berrocal, M. Colombo, P. Gallego, L. Seoane, C. Quintana, J. Zafra, J. Saiz, A. Santiago, A. Marin, C. Gordillo, D. Escribano, M. Sanz-Palomino. "How to improve a critical performance for an ExoMars 2020 Scientific Instrument (RLS). Raman Laser Spectrometer Signal to Noise Ratio (SNR) Optimization" European Planetary Science Congress, EPSC2017-1002-1, 11, (2017).

[10] F. Rull, S. Maurice, I. Hutchinson, A. Moral, C. Perez, C. Diaz, M. Colombo, T. Belenguer, G. Lopez-Reyes, A. Sansano, O. Forni, Y. Parot, N. Striebig, J. A. Rodriguez-Prieto, J. Medina, P. Gallego, R. Canchal, P. Santamaria, G. Ramos, J. L. Vago. "The Raman Laser Spectrometer for the ExoMars Rover Mission to Mars". Astrobiology 17, 627-654 (2017).

\section{Introducción}

El instrumento Raman Laser Spectrometer (RLS) forma parte de la carga útil del módulo Pasteur de la misión ExoMars de la Agencia Espacial Europea (ESA). Esta misión espacial para la exploración de Marte será lanzada en el año 2020 teniendo como principales objetivos el estudio superficial del planeta, la investigación del subsuelo y la caracterización del ambiente geoquímico en función de la profundidad del suelo al contar con un taladro capaz de alcanzar una profundidad de $2 \mathrm{~m}$ [1]. El instrumento RLS será el encargado de analizar el contenido geoquímico y la composición elemental de las muestras en polvo mediante espectroscopía Raman, técnica no destructiva que permite determinar la composición de los materiales sin una preparación previa y de forma inmediata [2].

Este instrumento está compuesto principalmente por tres unidades: un espectrómetro SPU (Spectrometer Unit), un cabezal óptico iOH (internal Optical Head) y una unidad de control electrónico ICEU (Instrument Control and Excitation Unit) que incluye un láser de excitación.

La muestra, previamente pulverizada, del subsuelo marciano es excitada con un láser de diodo de 532nm. El cabezal óptico tiene doble función, por un lado focaliza la luz láser en la muestra y por otro lado, es el encargado de recoger la señal Raman y llevarla hasta la entrada del espectrómetro a través de una fibra óptica multimodo de $50 \mathrm{~mm}$ de diámetro y 0.22 de apertura numérica. El SPU es la unidad óptica encargada de realizar la espectroscopía asegurando sus prestaciones en condiciones ambientales muy exigentes de temperatura y radiación espacial. Además a esta unidad se le imponen unas altas restricciones de masa $<<$ $900 \mathrm{~g}$ ) y volumen. El mayor reto en el diseño del espectrómetro reside en conseguir asegurar las prestaciones del mismo en las restrictivas condiciones de temperatura, volumen, masa y hostil ambiente de radiación espacial.

El diseño óptico del SPU está basado en una red holográfica de transmisión que actúa como elemento dispersor, siendo la primera vez que se emplea este elemento en una aplicación espacial de exploración planetaria [3]. La configuración óptica del SPU es modular, consistente en un subsistema colimador, un subsistema colector y la red de difracción. El sistema óptico es totalmente refractivo y ha sido diseñado para cubrir el rango espectral de interés científico de $533 \mathrm{~nm}$ a $676 \mathrm{~nm}$ con una resolución espectral de 0.12 $0.15 \mathrm{~nm} / \mathrm{px}$.

El subsistema colimador es el encargado de recoger la energía a través de la fibra óptica y colimar la luz que alcanza la red de difracción. Debe asegurar el elevado grado de colimación que se requiere para mantener el comportamiento acromático en todo el rango espectral de trabajo del espectrómetro. La red de difracción holográfica de volumen está grabada sobre gelatina dicromatada y cuenta con una eficiencia superior al $70 \%$ en todo el rango espectral de trabajo. El subsistema colector se encarga de colectar la energía dispersada por la red y focalizarla en el elemento detector basado en tecnología CCD.

La selección de materiales del diseño óptico tiene, por un lado, un gran impacto en la acromatización y atermalización del instrumento. Y por otro, en el nivel de madurez tecnológico TRL (Technological Readiness Level) que debe superar toda tecnología embarcada en un sistema espacial [4]. Esta madurez debe ser demostrada tanto a nivel unidad como de componentes y materiales. Todos los elementos ópticos del instrumento, lentes y red de difracción, han demostrado su compatibilidad en el ambiente relevante de la misión: temperaturas extremas, vacío y radiación espacial. Para ello se ha hecho uso tanto de la herramienta de predicción del comportamiento de materiales ópticos en ambiente espacial, como del protocolo de caracterización de materiales ópticos desarrollados en el Área de Óptica Espacial del INTA [5]. 
El desarrollo de este espectrómetro ha seguido la filosofía de modelos típica de toda aplicación espacial. A lo largo de las distintas fases de un programa espacial y de las distintas etapas de diseño, fabricación, integración y ensayos de un instrumento se van produciendo diferentes modelos de los subsistemas y sistema con la finalidad específica de ir progresando hasta llegar al modelo final o modelo de vuelo del instrumento que será el que realmente se lance.

Los distintos modelos se desarrollan para verificar una funcionalidad específica y por tanto no tienen por qué ser totalmente representativos del sistema que están modelizando, sino solamente de los aspectos de interés. Existen muchos modelos diferentes y dependiendo de consideraciones como el calendario, el coste, los requisitos de la misión, el nivel de madurez de la tecnología empleada, la confianza en los suministradores, instalaciones disponibles, riesgos tecnológicos..., entre otros factores, el número de modelos a desarrollar es diferente.

En el caso del espectrómetro SPU se han fabricado, integrado y caracterizado cuatros modelos fundamentales para demostrar las prestaciones y compatibilidad del espectrómetro. El primero de ellos fue un modelo de laboratorio o Breadboard (BB) que pretendía demostrar la viabilidad de la técnica con los diseños propuestos. El segundo de los modelos desarrollados fue el STM (Structural-Thermal Model) que pretendía verificar los modelos térmico y mecánico propuestos por los ingenieros y diseñadores del instrumento. El siguiente modelo de espectrómetro fue el EQM (Engineering Qualification Model), modelo dedicado al análisis de las prestaciones en rangos superiores a los operativos del instrumento. Y por último, se ha desarrollado el modelo FM (Flight Model) o de vuelo que es el que se incluirá en el rover que será enviado a Marte en 2020.

\section{Diseño óptico}

El diseño óptico del espectrómetro es totalmente refractivo y de transmisión. Está diseñado para trabajar en el rango espectral visible requerido de $533 \mathrm{~nm}$ a $676 \mathrm{~nm}$ alcanzando una resolución espectral de 0.12 $0.15 \mathrm{~nm} / \mathrm{px}$. La magnificación del instrumento es de 0.7X dedicando 3 pixeles en la resolución espacial.

Tiene una configuración modular formada por un colimador, que se encarga de colectar la energía procedente de la muestra excitada a través de una fibra óptica multimodo de 50 micras de diámetro y apertura numérica 0.22 y colimar la luz para llevarla hasta la red de difracción. El segundo módulo es el elemento dispersor, que dispersa espectralmente el flujo de energía recogido por el colimador. Esta luz dispersada es focalizada por el último módulo, el subconjunto colector, en el plano focal. La imagen final se forma sobre una línea del detector ocupando alrededor de 1100 pixeles equivalente a $17 \mathrm{~mm}$. El detector seleccionado es un dispositivo CCD-230-22 de e2v montado sobre un dispositivo termoeléctrico. El tamaño del pixel del detector es de 15 micras.

La figura 1 muestra el diseño óptico del espectrómetro SPU.

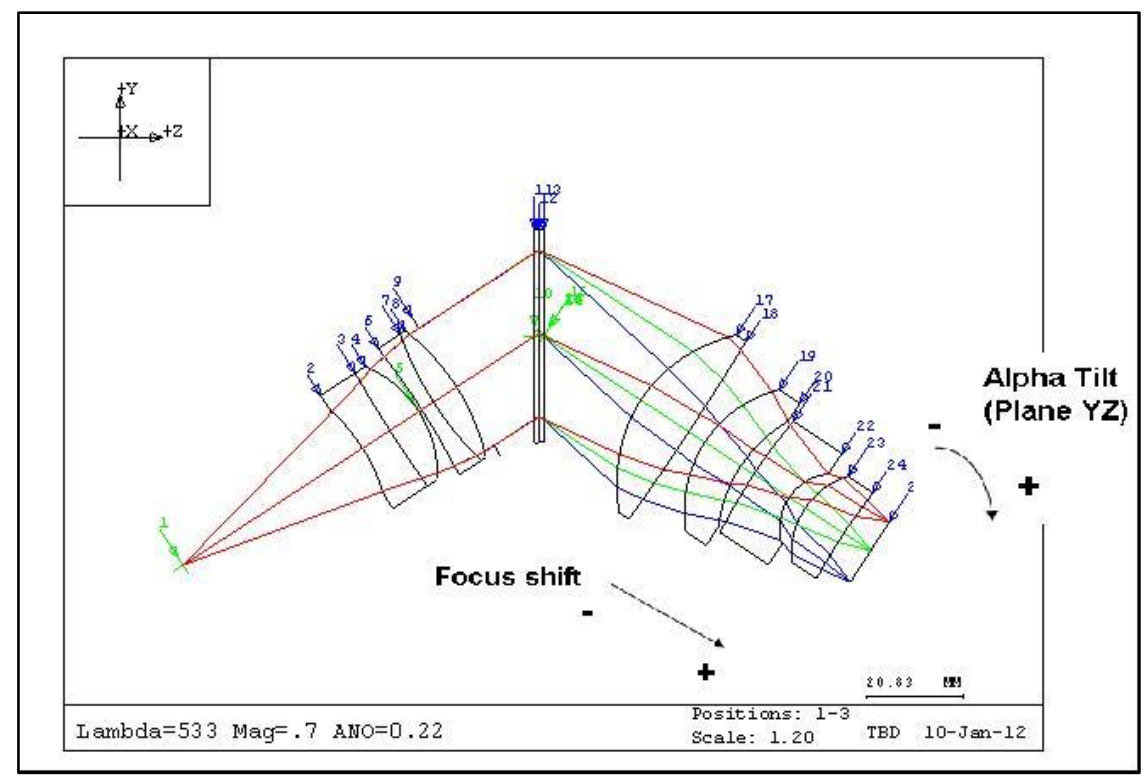

Fig 1. Diseño óptico del instrumento SPU para la exploración planetaria. 
El subsistema colimador está compuesto por dos dobletes (SF4, NFK5, NLAF2 de SCHOTT) y ha sido diseñado con la mejor calidad para mantener el grado de colimación acromático en todo el rango espectral de trabajo del espectrómetro y así asegurar el correcto funcionamiento del elemento dispersor. Presenta una longitud focal de $69.71 \mathrm{~mm}$ y con el fin de evitar pérdidas de energía del instrumento el número-f del colimador, f/\# 2.27, se ha adaptado a la apertura numérica de la fibra óptica de entrada.

El elemento dispersor es una red de difracción holográfica de volumen grabada sobre gelatina dicromatada y embebida entre dos vidrios de sílice fundida recubiertos por una capa antirreflejante y pegados con un adhesivo compatible con espacio como es el NOA61.

Esta red de difracción presenta $1800 \mathrm{l} / \mathrm{mm}$ y ha sido construida para la longitud de onda de $603 \mathrm{~nm}$. El ángulo de incidencia al que trabaja esta red y el ángulo de difracción es el mismo, $32.84^{\circ}$, proporcionando una eficiencia de difracción superior al 70\% en todo el rango espectral de trabajo del espectrómetro (Figura 2). La escasa información proporcionada por el suministrador [6] sobre el comportamiento óptico de las redes de difracción en ambiente espacial obligó a efectuar una caracterización previa del dispositivo y validar su compatibilidad en espacio [3].

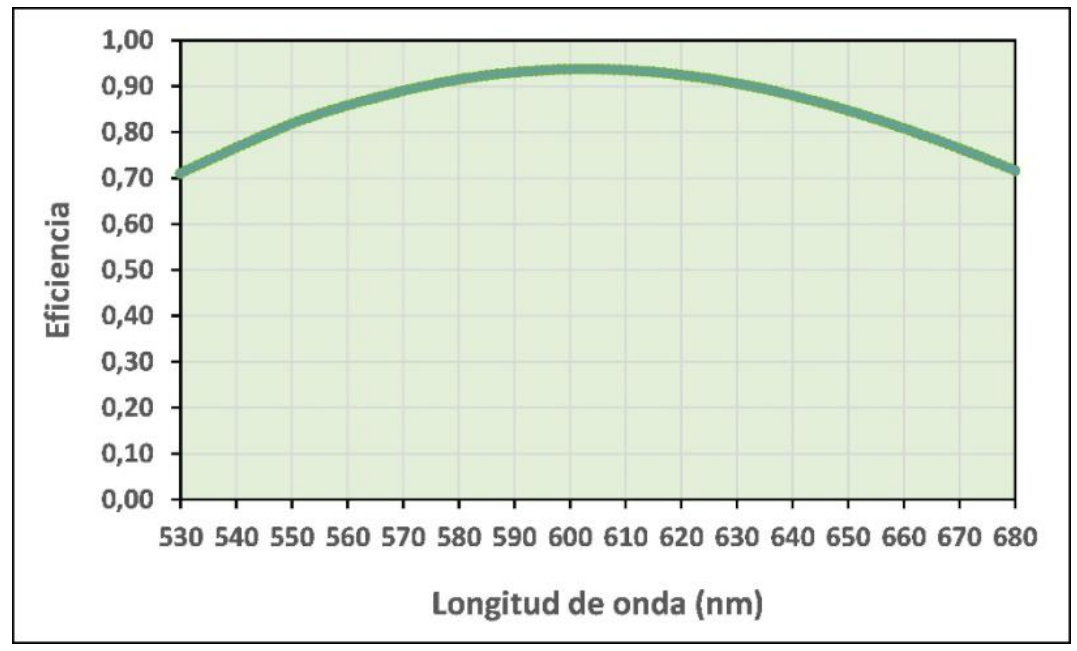

Fig 2. Eficiencia de la red holográfica de volumen del SPU.

El tercer subsistema del SPU es el colector. Este conjunto está compuesto por cuatro lentes, tres de ellas esféricas, una de NLAK22 y dos de SF4 de SCHOTT, y una lente cónica, NBALF4, del mismo fabricante. La longitud focal del colector es $48.80 \mathrm{~mm}$ y su número-f de 1.59 que permite tener margen en el acople con el subsistema anterior. Adicionalmente este subsistema presenta un descentramiento angular de $-0.784^{\circ}$ con respecto a la incidencia normal de la longitud de onda central, usada como referencia, para compensar los órdenes de difracción en el rango espectral requerido y obtener las mejores prestaciones ópticas. Con el fin de controlar el tamaño de este subsistema se coloca un stop de apertura de $30 \mathrm{~mm}$ antes de la red de difracción asegurando un diseño óptico más compacto.

El procedimiento seguido para la optimización del diseño óptico del espectrómetro SPU fue diseñar y optimizar sus prestaciones en condiciones operativas del instrumento, es decir a una temperatura de $-20^{\circ} \mathrm{C}$, temperatura media del rango térmico en el que trabaja el espectrómetro $\left(-40^{\circ} \mathrm{Ca}+6^{\circ} \mathrm{C}\right)$, y presión marciana $(6.002 \mathrm{mmHg})$. Esta evaluación se realizó teniendo en cuenta que el material seleccionado para separadores, anillos de retención y estructura mecánica del espectrómetro era de titanio, material con un coeficiente de expansión térmico similar al de los vidrios $\left(8.6 \times 10^{-6} /{ }^{\circ} \mathrm{C}\right)$.

El diseño optimizado del SPU fue una alternativa entre las mejores prestaciones en condiciones ambientales (condiciones de integración del instrumento) y las mejores prestaciones en condiciones operacionales del instrumento (ambiente marciano). Esta situación implicó un desenfoque de $\sim 94 \mu \mathrm{m}$ del plano imagen en condiciones ambientales para compensar el golpe de vacío y las variaciones térmicas.

\section{Selección de materiales}

La selección de materiales ópticos para el diseño se ha realizado teniendo en cuenta el impacto en la acromatización y atermalización del instrumento. El caso ideal reside en la selección de unos pares de 
vidrios que compensen ambas aberraciones, la cromática y la sensibilidad a los cambios de temperatura. En una primera aproximación se calculó el cambio de focal de los dobletes con la longitud de onda y la temperatura siguiendo el procedimiento clásico [7] para realizar esta compensación cumpliéndose la siguiente realización (1):

$$
V_{A} \cdot \varepsilon_{A}=V_{B} \cdot \varepsilon_{B}
$$

donde $V$ es el número Abbe de cada vidrio del doblete y $\varepsilon$ se expresa para cada material como

$$
\varepsilon=\left(\frac{1}{(n-1)} \cdot \frac{d n}{d T}\right)-\alpha
$$

siendo $\alpha$, el coeficiente térmico de cada vidrio.

Por otro lado, como ya se ha comentado, la selección de los materiales ópticos también tiene impacto en el nivel de madurez tecnológico (TRL) [4] que debe alcanzar el espectrómetro para ser embarcado. El comportamiento de las propiedades ópticas de los mismos como cambios en el índice de refracción, pérdida de transmitancia, degradación superficial, coloración..., muestra una fuerte dependencia con las condiciones espaciales en las que se encuentran [8]. El conocimiento previo de este comportamiento facilita a los diseñadores ópticos la selección de materiales que mejor se adapten a los requisitos de vida de la misión pudiendo de esta forma predecir y valorar la influencia de las pérdidas de prestaciones ópticas de la instrumentación antes de la puesta en órbita del instrumento.

En el Área de Óptica Espacial del Instituto Nacional de Técnica Aeroespacial (INTA) se ha desarrollado, por un lado, una herramienta software capaz de predecir la pérdida de propiedades ópticas de un material, contando con una amplia base de datos, así como un protocolo de caracterización de la influencia de las condiciones de ambiente espacial en las propiedades ópticas de los materiales [5]. Para la selección de los materiales ópticos utilizados en el diseño de este espectrómetro se ha hecho uso tanto del programa de predicción como del protocolo de caracterización de materiales ópticos.

\section{Resultados}

El objetivo principal del espectrómetro SPU es alcanzar la resolución necesaria para resolver los picos procedentes de la señal Raman en las condiciones ambientales impuestas por la misión, vacío, temperaturas extremas y radiación espacial, así como la relación-señal-ruido (SNR, Signal-to-Noise Ratio) necesaria para distinguir en un espectro Raman posición, número e intensidad de los picos que caracterizan la fase y composición de un material [9]. Además, dicho instrumento también ha de cumplir con un mínimo de calidad óptica evaluada en térmicos de MTF (Figura 3) y RMS (Figura 4).

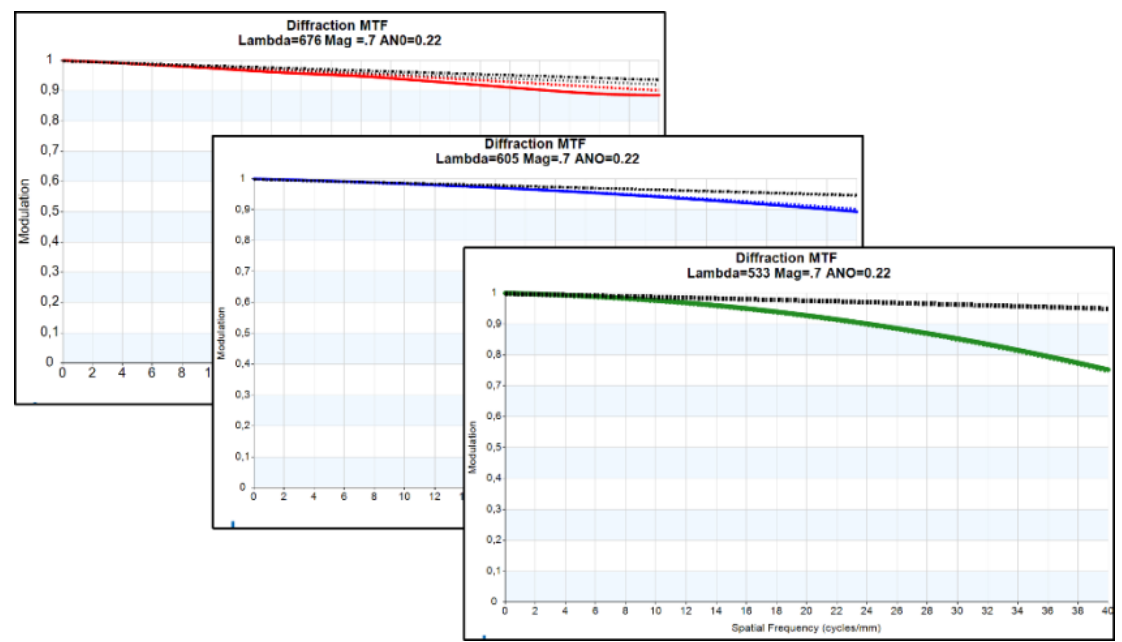

Fig 3. Curvas de MTF para las longitudes de onda extremas y central en el rango de trabajo del espectrómetro. 

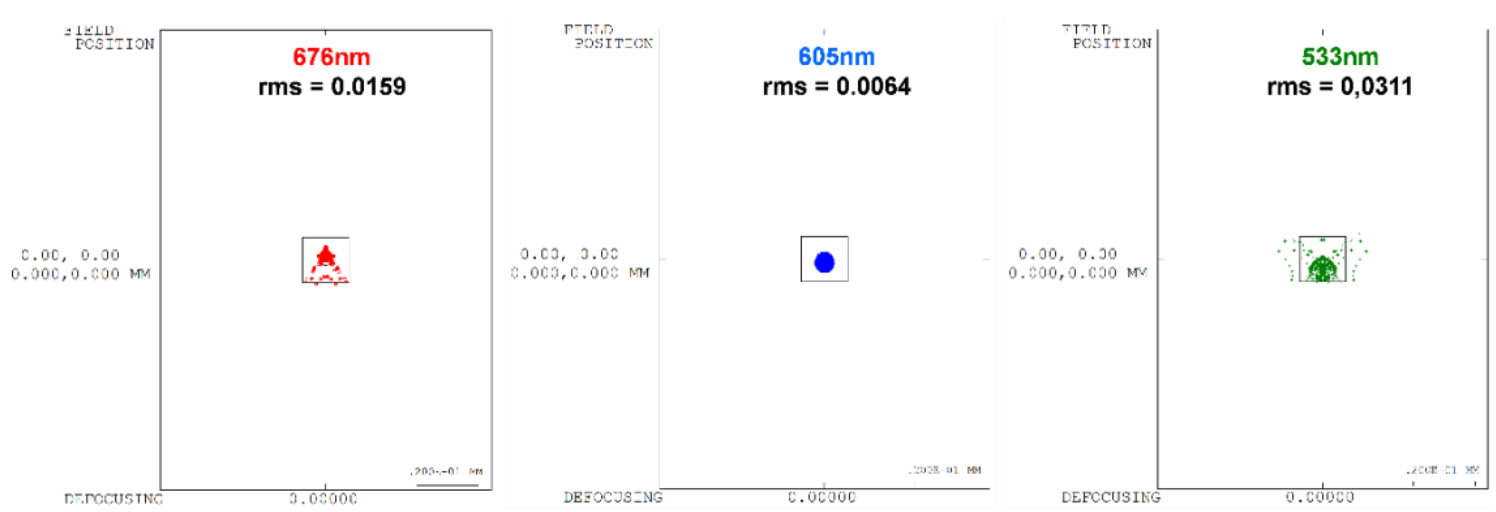

Fig 4. Spot diagram para las longitudes de onda extremas y central en el rango espectral de trabajo del espectrómetro. El cuadro central representa el tamaño del pixel, $15 \mu \mathrm{m}$

La MTF fue seleccionada como la figura de mérito para analizar las prestaciones dado que el INTA cuenta con un banco óptico dedicado a ello y por ser una medida relativamente fácil de realizar en el laboratorio. La frecuencia de Nyquist del sistema (33lineas $/ \mathrm{mm}$ ) fue la frecuencia espacial de referencia en la cual la MTF fue verificada. Esta verificación se realizó a nivel de subsistema, es decir, se midió la calidad óptica de cada uno de los conjuntos que componen el espectrómetro, el subsistema colimador y el subsistema colector. La siguiente tabla muestra los valores de la MTF del colimador y colector para la frecuencia de Nyquist en función de tres longitudes de onda en el rango de trabajo del espectrómetro, 530nm, $600 \mathrm{~nm}$ y $670 \mathrm{~nm}$.

TABLA 1. MTF en función de la longitud de onda y para la frecuencia de Nyquist (33l/mm).

\begin{tabular}{|c|c|c|c|}
\hline \hline Subsistema & $\begin{array}{c}\text { Longitud de onda } \\
(\mathrm{nm})\end{array}$ & $\begin{array}{c}\text { MTF } \\
\text { diseño+tolerancias }\end{array}$ & $\begin{array}{c}\text { MTF } \\
\text { experimental }\end{array}$ \\
\hline \hline & 670 & $0.80 \pm 0.08$ & $0.89 \pm 0.01$ \\
Colimador & 600 & $0.73 \pm 0.07$ & $0.88 \pm 0.01$ \\
& 530 & $0.53 \pm 0.05$ & $0.71 \pm 0.01$ \\
\hline \hline & 670 & $0.76 \pm 0.08$ & $0.68 \pm 0.01$ \\
Colector & 600 & $0.72 \pm 0.07$ & $0.65 \pm 0.01$ \\
& 530 & $0.70 \pm 0.07$ & $0.62 \pm 0.01$ \\
\hline
\end{tabular}

La resolución espectral definida como la separación mínima entre dos longitudes de onda próximas que nos permite distinguir ambas longitudes de onda fue verificada a través de la medida de la dispersión lineal que proporciona el espectrómetro. La siguiente tabla muestra, en la segunda columna, la dispersión lineal que por diseño óptico proporciona el espectrómetro y en las últimas columnas la dispersión lineal y resolución espectral equivalente medida en el laboratorio.

TABLA 2. Dispersión lineal y resolución espectral en función de la longitud de onda.

\begin{tabular}{|c|c|c|c|}
\hline \hline $\begin{array}{c}\text { Longitud de onda } \\
\text { (nm) }\end{array}$ & $\begin{array}{c}\text { Dispersión lineal } \\
\text { (nm/mm) }\end{array}$ & $\begin{array}{c}\text { Dispersión lineal } \\
\text { experimental (nm/mm) }\end{array}$ & $\begin{array}{c}\text { Resolución espectral } \\
\text { (nm/pixel) }\end{array}$ \\
\hline \hline 537.16 & 9.67 & $9.86 \pm 0.06$ & 0.148 \\
551.98 & 9.82 & $9.84 \pm 0.06$ & 0.148 \\
580.84 & 9.84 & $9.82 \pm 0.06$ & 0.147 \\
601.81 & 9.65 & $9.78 \pm 0.06$ & 0.147 \\
609.15 & 9.55 & $8.88 \pm 0.06$ & 0.133 \\
620.48 & 9.37 & $9.58 \pm 0.06$ & 0.144 \\
632.25 & 9.12 & $8.86 \pm 0.06$ & 0.133 \\
657.18 & 8.42 & $7.94 \pm 0.06$ & 0.119 \\
675.74 & 7.69 & $7.94 \pm 0.06$ & 0.119 \\
\hline
\end{tabular}


La verificación de las prestaciones como instrumento electro-óptico se realizó a través de la medida de la SNR, relación entre el nivel de señal colectado por el detector del instrumento y el nivel de ruido generado en el mismo. En la columna tercera de la Tabla 3 se muestra el requisito de SNR impuesto por la misión teniendo en cuenta el ambiente en el que opera el instrumento y el tiempo de integración máximo para adquirir la señal Raman. En la cuarta columna de la misma tabla aparece el valor de SNR medido con el espectrómetro de vuelo.

TABLA 3. SNR alcanzada por el espectrómetro en función de la longitud de onda correspondientes a los distintos picos Raman de materiales de referencia.

\begin{tabular}{|c|c|c|c|}
\hline $\begin{array}{c}\text { Material de } \\
\text { referencia }\end{array}$ & $\begin{array}{c}\text { Pico fundamental } \\
\text { (nm) }\end{array}$ & Requisito SNR & $\begin{array}{c}\text { SNR } \\
\text { experimental }\end{array}$ \\
\hline \hline Silice & 546 & 250 & 1868 \\
\hline \hline Ciclohexano & 551 & 1500 & 1868 \\
\hline \hline Calcita & 564 & 1188 & 1888 \\
\hline Calcita & 552 & 65 & 418 \\
\hline \hline Calcita & 575 & 16 & 225 \\
\hline Calcita & 586 & 14 & 251 \\
\hline \hline Hematita & 543 & 38 & 207 \\
\hline
\end{tabular}

La filosofía de modelos seguida para el desarrollo de este instrumento de aplicación espacial permitió demostrar tanto las prestaciones como la compatibilidad del espectrómetro. El modelo de laboratorio BB, permitió verificar la funcionalidad del SPU (Figura 5.1), es decir si cumplía con la resolución espectral y relación señal-ruido (SNR) requeridas. Con el segundo modelo STM se verificaron los modelos analíticos térmico y estructural, se validó el diseño termo-mecánico y la viabilidad de la fabricación. Este modelo (Figura 5.2) fue sometido a pruebas de carga, choque y balance térmico sin llevar componentes ópticos. El siguiente modelo de espectrómetro fue el EQM, éste es totalmente representativo de vuelo y está fabricado con componentes de vuelo (Figura 5.3). Este modelo se sometió a ensayos ambientales (térmicos, mecánicos a nivel unidad y de compatibilidad electromagnética EMC a nivel instrumento) hasta niveles de calificación, es decir niveles límites, para garantizar su supervivencia y correcto funcionamiento. Y por último se ha desarrollado el modelo FM o de vuelo (Figura 5.4), exactamente igual que el modelo EQM pero sometido a ensayos de aceptación en los que los esfuerzos ambientales son limitados al supuesto peor caso en las condiciones de vuelo.

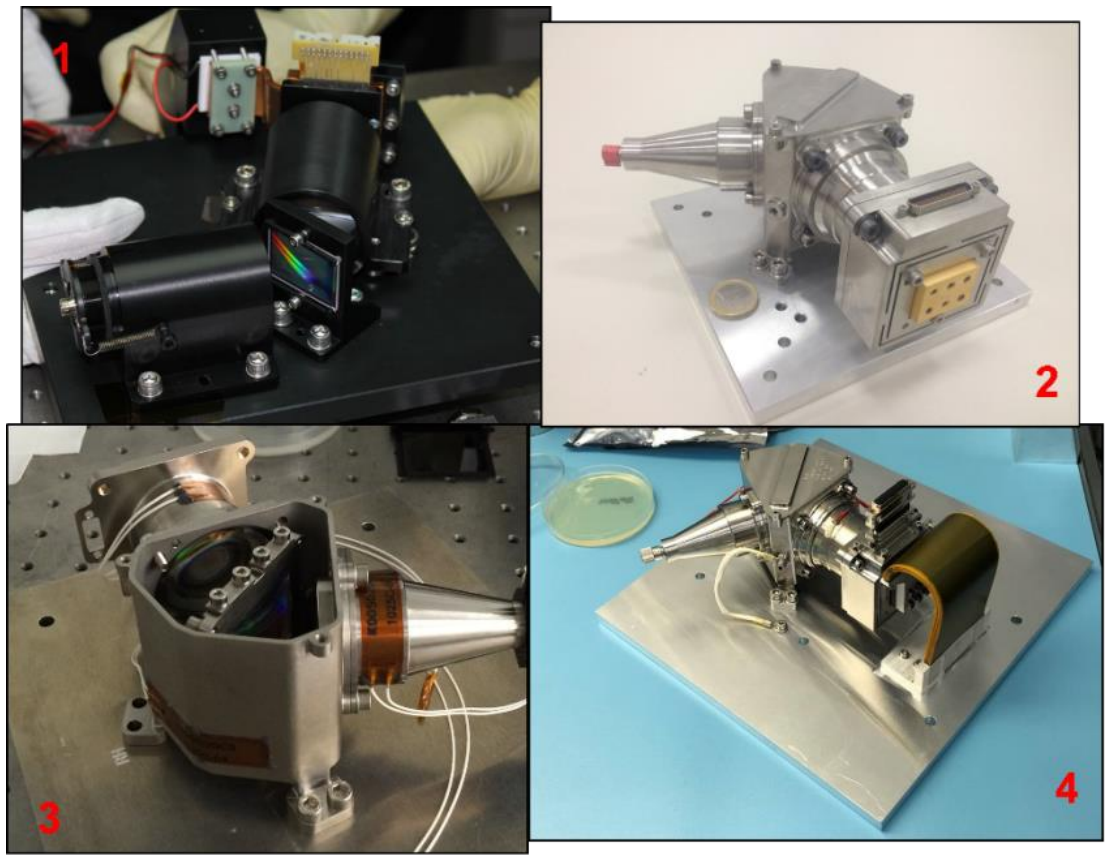

Fig 5. Modelos desarrollados del espectrómetro: 1) BB, 2) STM, 3) EQM y 4) FM. 
Tanto la resolución espectral como la relación señal-ruido, prestaciones ópticas requeridas al instrumento, fueron verificadas en las distintas condiciones ambientales en las que se espera que opere el instrumento.

La siguiente figura muestra la imagen en el CCD de todo el rango espectral de trabajo del espectrómetro (izquierda) y del espectro de una lámpara de calibración de Ne (derecha). Ambas imágenes fueron tomadas con el modelo de vuelo del espectrómetro y permitieron verificar el cumplimiento de los requisitos de resolución espectral y SNR que proporcionan al espectrómetro la capacidad de identificar la composición de materiales en condiciones de temperatura y radiación extremas como las que soportará el instrumento RLS en Marte [10].

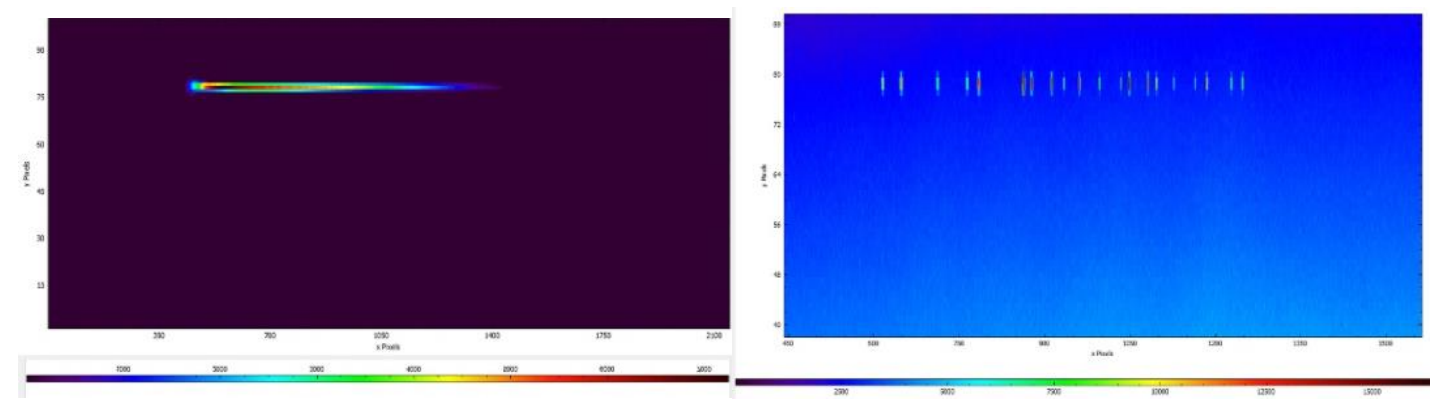

Fig 6. Imágenes tomadas con el SPU de vuelo: fuente de luz blanca (izquierda) y de una lámpara de calibración de Ne (derecha).

\section{Conclusiones}

El espectrómetro con magnificación 0.7X traslada la fibra óptica al plano imagen del detector alcanzando la resolución necesaria $(0.12-0.15 \mathrm{~nm} /$ pixel) para resolver los picos Raman separados entre $0.17-0.37 \mathrm{~nm}$, dependiendo de la zona del rango espectral visible, y cuenta con la capacidad para colectar el flujo de señal necesario en la detección de dichos picos (throughput óptico $>0.5$ ). La calidad del instrumento fue evaluada y verificada en términos de MTF mostrando una calidad equivalente a $\lambda / 4$, alta calidad para un diseño óptico no formador de imagen.

La selección de materiales (lentes, recubrimientos y red de difracción) aseguran las prestaciones del instrumento en término de resolución y de SNR en el ambiente espacial de la misión.

El desarrollo de los distintos modelos dentro de la filosofía de instrumentación espacial, ha permitido llegar a la realización del modelo de vuelo del espectrómetro que será embarcado en el rover de la misión ExoMars en el año 2020 alcanzando las prestaciones requeridas y cumpliendo con las restricciones de masa y volumen.

\section{Agradecimientos}

Partes de este trabajo fueron presentadas en la XII Reunión Nacional de Ópticas en “Diseño Óptico de un Espectrómetro de Altas Prestaciones para la Exploración Planetaria”, 2018. Los autores agradecen al Ministerio de Economía, Industria y Competitividad (MINECO) la financiación recibida para la contribución española en este programa (ESP2014-56138-C3-1R). 